\title{
Cómo orientar la formación del personal docente nuevo hacia la adquisición de un paradigma sistémico
}

\author{
Guiding New Teacher Training towards the Acquisition of a Systemic Paradigm

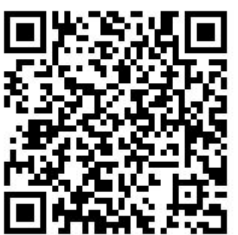 \\ Luis Guillermo Barrantes-Montero' \\ Universidad Nacional \\ Escuela de Literatura y Ciencias del Lenguaje \\ Heredia, Costa Rica \\ luisba66@yahoo.es
}

Recibido 14 de noviembre de 2014 • Corregido 5 de agosto de 2015 • Aceptado 14 de agosto de 2015

Resumen. Mediante una distinción entre el paradigma disciplinario positivista, que segmenta el saber humano en disciplinas aisladas y el paradigma sistémico, que busca la integración del conocimiento con el recurso a la inter y transdisciplinaridad, se señala la conveniencia y necesidad de preparar al personal docente nuevo en este último. Se expone un ejemplo de cómo realizar la experiencia formativa según el paradigma sistémico en un curso de Lectura avanzada en inglés, en una carrera de formación de docentes.

Palabras claves. Interdisciplinaridad, transdisciplinaridad, educación superior, paradigma.

Abstract. This paper distinguishes between positivist disciplinary paradigm, which classifies human knowledge into isolated disciplines, and systemic paradigm, which aims at integrating knowledge and an inter-and trans-disciplinarity approach. The need and convenience of preparing new professionals in the latter is discussed. An example on how to develop a training experience based on the systemic paradigm in an advanced English reading course, in a teacher-training career is also described.

Key words: Interdisciplinarity, transdisciplinarity, higher education, paradigm.

\footnotetext{
${ }^{1}$ Posee una maestría profesional en la Enseñanza del Inglés por la Universidad de Costa Rica (2006). Cuenta, además, con una licenciatura en Traducción Inglés-Español por la Universidad Nacional, Costa Rica (1996), una licenciatura en Teología por la Universidad Francisco Marroquín, Guatemala (1992) y un bachillerato en Filosofía por la Universidad Francisco Marroquín, Guatemala (1989). Durante diecinueve años se ha desempeñado como docente de inglés y de español como segunda lengua. Actualmente coordina el Departamento de Cursos de Servicio en la Universidad Nacional, Costa Rica. Ha realizado ponencias en congresos internacionales y varios de sus artículos y ponencias han sido publicados en revistas como EDUCARE y LETRAS, ambas de la Universidad Nacional.
} 
doi: http://dx.doi.org/10.15359/ree.19-3.22

URL: http://www.una.ac.cr/educare

CORREO: educare@una.cr

El quehacer académico de la Universidad plantea la articulación permanente entre diversas disciplinas y la búsqueda de su complementariedad

Universidad Nacional, Costa Rica (2014, p. 2)

\section{Introducción}

Dentro de la acción sustantiva de la educación superior, el proceso de formación docente reviste una singular importancia, ya que estos nuevos profesionales van a tener una incidencia directa y quizás definitiva en las generaciones jóvenes de la sociedad (UNESCO, 2002, citado por Asencio, 2013.

Resulta lamentable, y esto por el testimonio vivo y reiterado de gran parte de la población estudiantil universitaria, que en no pocas de las carreras que cursan se da por sentado que el paradigma epistémico por el que se rigen, se ajusta de manera automática al conjunto de valores y premisas que les presenta, como dice Hahn (2003) "the changing Zeitgeist" [el cambiante espíritu de los tiempos] (p.199). Incluso, tal vez más grave aún, que para algunos las nociones mismas de paradigma y matriz epistémica les resulten desconocidas 0 , cuando menos, ajenas a su supuesta especialidad.

Este trabajo plantea una preocupación real y concreta del autor por el rumbo de la formación de docentes del siglo XXI en las instituciones universitarias. Se propone y defiende la firme aserción de que las nuevas generaciones de docentes deben formarse mediante el paradigma de la complejidad (Morin, 2007), es decir, con un enfoque inter y transdisciplinario, el cual asuman como propio en sus vidas y lo transmitan a sus educandos.

Se contribuye con un ejemplo bastante somero de la aplicación de este enfoque; se espera, con ello, suscitar el interés por ahondar en este tema. Con ocasión del recién celebrado Congreso Universitario de la Universidad Nacional, Costa Rica (2014), en el cual se modifica su Estatuto Orgánico, se busca ofrecer luces para la consecución de las metas que ahí se proyectan.

\section{La urgencia de una transformación paradigmática}

Los avances científicos y tecnológicos en este tercer milenio suelen darse con una frecuencia y rapidez de tal magnitud que a las sociedades mismas, e incluso a los aparatos institucionales que las gobiernan, les resulta difícil ajustarse a ellos y mantenerse actualizados (Blázquez, 2001). Si esta realidad es patente en las naciones líderes en investigación científica e innovación tecnológica, cuanto más lo será en aquellas que, fundamentalmente, dependen de la transferencia de tecnologías y de los llamados datos de prueba de los centros de vanguardia en investigación para poder desarrollar, a partir de ellos, versiones locales de los adelantos que, de un modo u otro, puedan beneficiarlas. 
Desde el Renacimiento Carolingio, los centros de producción y articulación del conocimiento, sobre todo las universidades, por propósitos metodológicos, organizaron los campos de estudio con base en una celosa delimitación de los ámbitos de cada disciplina o campo del saber. Esa intención que, en principio, era legítima, llevó a la academia a un excesivo celo por los distintos campos profesionales.

El paradigma uni-disciplinario o mono-disciplinario (Martínez, 2009) devino, entonces, de índole inductiva, aislacionista y reduccionista. Su consecuencia más inmediata fue el sesgo en la formación de los nuevos profesionales, al no prepararlos para la integración del conocimiento -tanto en el dominio cognitivo como en el afectivo-, no se les enseñó a aprender a aunar la objetividad con la subjetividad y la mismidad con la otredad (Lynch, Russell, Evans y Sutterer, 2009). Una carrera universitaria consiste, para ellos, según eso, en completar satisfactoriamente una serie de cursos con una mínima afinidad intrínseca, pero desprovistos de toda relación o sinapsis con las demás dimensiones que componen ser en cuanto personas humanas, insertas en la historia y sujetas a todas las contingencias de tiempo y contexto.

En vista de lo anterior, urge que la preparación de nuevos docentes sea sometida a una transformación paradigmática, un abordaje de la articulación de los conocimientos que opere en modo ascendente, desde la simplificación hasta la complejidad (Roa, 2006). Urge pasar de la visión atomística y monadológica a una visión sistémica. El paradigma sistémico es el que puede llevar a las nuevas generaciones de docentes a trascender el paradigma disciplinario y abocarse con entusiasmo en un nuevo paradigma de corte inter y transdisciplinar.

\section{El enfoque inter y transdisciplinario en el proceso de formación de docentes}

Iniciar a los docentes y las docentes en formación en un proceso de transformación paradigmática hacia un modelo sistémico de inter y transdisciplinaridad implica que, de ser posible, las unidades académicas sometan a revisión los planes de estudio y los programas de curso de sus carreras, para que en ellos se refleje la manera concreta en que los contenidos del respectivo campo del saber serán abordados con miras hacia la complejidad. Como lo expresa Roa, 2006, citando a López, 1997:

Frente al precepto de la evidencia, la complejidad asume la incertidumbre; frente al precepto de la fragmentación, la complejidad [asume] que el todo es más (o menos) que la suma de sus partes aisladamente consideradas; ... frente al precepto de la exhaustividad, la complejidad asume la esencial incompletud del conocimiento y el papel de nuestras representaciones como aproximación limitada y parcial a lo real. (p. 151) 
doi: http://dx.doi.org/10.15359/ree.19-3.22

URL: http://www.una.ac.cr/educare

CORREO: educare@una.cr

El efecto multiplicador de este enfoque de formación se hará patente cuando estos formandos, una vez insertos en el mundo de la docencia, contribuyan en la creación de un talante integrador de conocimientos en las nuevas generaciones.

La intención de asumir el paradigma de la complejidad en la formación docente consiste en rectificar el modo de ser y de actuar de la sociedad mediante la educación de la población joven. Ya se probó la represión y no hubo buenos resultados; tampoco se lograron mejores condiciones mediante la espontánea segregación de los grupos marginales y la creación de ciudades privadas para los estratos privilegiados. Cansadas del agotamiento social, económico, político y ecológico, las naciones deben enrumbar sus sistemas educativos hacia la formación de una ciudadanía que se conciba a sí misma y su ser-en-el-mundo como parte integrante y vital de sistemas interconectados, que ningún individuo puede lograr su realización plena al margen de su responsabilidad colectiva.

\section{Ejemplo de iniciación de docentes en la formación de una Weltanschauung inter y transdisciplinaria}

Una vez planteado el problema e ilustrado mediante una brevejustificación teórica, conviene presentar un ejemplo simple de cómo se puede operativizar esta preocupación por hacer de las aulas universitarias recintos de reflexión donde las experiencias subjetivas del estudiantado cuenten tanto como los contenidos expuestos por el personal docente y los insumos didácticos que sustenten el aprendizaje, lo que Tudor (2000) llama la ecología de la educación.

El curso de lectura avanzada, componente de la carrera de bachillerato en primer y segundo ciclos con énfasis en la enseñanza del inglés de la Universidad Nacional de Costa Rica en el segundo ciclo 2014, se propuso dos metas globales para el estudiantado, dentro del marco de objetivos contemplados en el programa de curso:

1. Mostrar evidencia de pensamiento crítico en intervenciones orales y asignaciones escritas mediante la aplicación de estrategias y pautas de organización de ideas provistas por el docente.

2. Establecer nexos e implicaciones del material de lectura extensiva con otros ámbitos del saber y con la cotidianidad del estudiantado mediante la aplicación del enfoque inter, transdisciplinar.

Una de las fuentes de lectura extensiva en el curso fue la novela, en su versión original en inglés, de Brown (2004) The Da VinciCode [Elcódigo Da Vinci]. La novela se caracteriza por la referencia a eventos y lugares históricos de gran relevancia para la cultura general del estudiantado, lo que obliga a contar con fuentes de referencia que expliquen cotejen o complementen la información presentada. Además, se entretejen diversas tramas que no escapan a la controversia, sobre todo de índole religiosa. Cabe destacar, también, que el autor juega ingeniosamente con datos verídicos e historias ficticias, lo que demanda una aguda criticidad por parte de quienes leen la obra. 


\section{A continuación se presenta un resumen del proceso llevado a cabo por el alumnado con} la guía del docente.

\section{Etapas del proceso de lectura (Douglas, Huntley y Rogers, 2012)}

1. La pre-lectura: Una vez seleccionado el texto y en aplicación de las estrategias que se describen a continuación, se procura evocar tanto conocimiento previo acerca del tema como sea posible. También se identifican las posibles fuentes de información que ayudarán a despejar dudas o enriquecer informaciones.

2. La lectura: A medida que se avanza en la lectura se toma nota de detalles o aspectos de relevancia y que requieren de clarificación o profundización posterior.

3. La post-lectura: Se recurre a las fuentes complementarias para investigar acerca de los detalles o aspectos señalados durante la lectura y se prepara el modo de compartirlo con las demás personas de la clase.

Estrategias generales de lectura (Soto, I. Villalobos y L. Villalobos, 2007)2

1. Visión de conjunto del texto. (Skimming) Es una visión rápida y no detallada del texto en el que se busca reconocer su organización, divisiones y subdivisiones y temática general.

2. Escaneo de información específica. (Scanning) Se identifican detalles específicos, personajes, datos o eventos que pueden o deben ser objeto de cotejo, análisis o profundización con otras fuentes.

3. Distinción entre hecho y opinión. Es fundamental que la persona que lee atienda la diferencia entre hechos y opiniones emitidas por el narrador de la novela (en este caso) o sus personajes.

4. Distinción entre ideas principales y secundarias. En cada capítulo de la novela se desarrolla un tema en torno a una idea principal, la cual es apoyada por ideas secundarias. Al analizar críticamente el texto, quienes leen deben saber identificar unas y otras.

5. Parafraseo. Al hacer referencia a lo dicho por el narrador o los personajes de la novela, es de suma importancia contar, no solo con la comprensión del texto, sino también con el vocabulario y cohesión léxica y gramatical para evitar el uso de las repeticiones textuales del original.

6. Inferencia. Quien lee, debe ir más allá de lo que el texto propone de manera explícita para llegar a conclusiones propias. Esto será clave en el análisis crítico.

${ }^{2}$ Aunque existen muchas otras estrategias de lectura, en el trabajo que nos ocupa se desarrollaron con especial interés las enumeradas en el cuadro. 
doi: http://dx.doi.org/10.15359/ree.19-3.22

URL: http://www.una.ac.cr/educare

CORREO: educare@una.cr

Estrategias de pensamiento crítico (Sadeghi, Taghi y Rahmatkhah, 2014)

1. Cotejo de informaciones acerca de personajes y eventos. La novela en cuestión combina la aparición de personajes ficticios con figuras de relevancia histórica. También se presentan eventos o entidades que deben ser cotejadas en fuentes complementarias.

2. Ampliación de informaciones. Aquellas informaciones que cuentan con sustento histórico requieren de un tratamiento de profundización especial con la finalidad de instruirse en cuanto a su tiempo, lugar y contexto y comprender la razón de su referencia en el texto.

3. Uso de la intertextualidad. En el caso de The Da VinciCode, resulta de suma trascendencia el descubrir elementos intertextuales con otras obras o incluso con las mismas fuentes bíblicas a las que se alude continuamente.

4. Aplicación del conocimiento previo. Todo lo que los lectores saben acerca de los temas, subtemas, lugares, figuras y eventos mencionados en el texto cobra relevancia a la hora de establecer relaciones $y$, sobre todo, al hacer análisis críticos.

5. Confrontación de planteamientos del texto con puntos de vista propios. Para el estudiantado joven, no deja de ser algo intimidante el que se les solicite aportar sus propias contribuciones al debate, tomar posición frente a enunciados y expresar puntos de vista propios si en su trasfondo de escolaridad previa tuvieron poca exposición a estas metodologías. Sin embargo, una vez encaminados a manifestarse con libertad, les resulta no solo gratificante sino también y estimula su anhelo de investigación.

\section{Estrategias de inter y transdisciplinaridad (Martínez, 2010)}

1. Interdisciplinaridad: Relaciones entre disciplinas de modo que estas respondan a los objetivos de enseñanza (Oliva Calvo). La novela que se ha escogido para este estudio contiene un potencial interdisciplinar muy a propósito para los objetivos que perseguimos. Al adentrarse en la trama de los capítulos, los lectores identifican personajes, eventos y datos de relevancia y los van asociando con campos del saber que les son pertinentes. La primera constatación por parte de los estudiantes es que la manera óptima de aprender acerca de algo es tendiendo hacia su complejidad en vez de hacia su simplificación. Al abordar los temas de estudio de forma holística y sistémica, se descubren repercusiones cada vez más cautivantes, lo que no se lograría desde un marco epistemológico de corte positivista.

2. Transdisciplinaridad: Interacción recíproca de los saberes de campos dispares con el fin de establecer conexiones de relación y subordinación (Oliva Calvo). En el caso de la novela que nos ocupa, la experiencia interdisciplinar del grupo de estudiantes, comienza con una identificación de elementos que tocan los campos de acción de disciplinas dispares: Historia (de la Iglesia, del arte, del Renacimiento), Teología, Simbolismo religioso, Arte renacentista, etc. Cada personaje, dato o evento que se menciona en los diferentes capítulos es investigado en fuentes específicas de cada campo. De este modo, por ejemplo, lo que aporta la Historia de la Iglesia con respecto al Arte Renacentista y a la connotada figura de Leonardo Da Vinci se confronta con lo que por su parte revelan los estudios sobre técnicas de arte y los movimientos artísticos del Renacimiento. $O$ bien, la visión de la feminidad desde la perspectiva bíblicoteológica, e incluso eclesiológica, se contrasta con lo que aportan la sociología y la antropología.

El procedimiento consiste en investigar los objetos de interés desde la posición de cada campo del saber para luego intentar una síntesis lo más balanceada posible en cada caso. 


\section{Los resultados}

Al llegar al final de la lectura de la primera mitad de la novela, las personas participantes de la clase realizaron presentaciones orales acerca del contenido de los correspondientes capítul os asignados. Las presentaciones consistían en una breve síntesis del contenido de la trama y, luego, una parte más sustancial acerca de los elementos de particular relevancia que debían ser profundizados con criterios de inter y transdisciplinaridad. Lo mismo tuvo lugar al completarse la lectura de la segunda mitad del libro.

Ciertamente, el disfrute pleno de la novela requiere de una profundización adyacente de múltiples elementos de campos especializados. Más aún, una lectura desprovista de esa ilustración complementaria para asumir los contenidos con pensamiento crítico, podría tener efectos contraproducentes como sesgos que conduzcan a prejuicios y malas interpretaciones de los hechos, instituciones o personajes que aparecen en el texto. La lectura de EI Código Da Vinci requiere, pues, un ejercicio de inter y transdisciplinaridad.

Ejemplos de los temas abordados son:

a) De la primera parte: La figura de Leonardo Da Vinci. A partir del capítulo 6, Da Vinci es referido en múltiples ocasiones por el autor de la novela, pues la trama involucra una serie de acertijos, mensajes secretos y elementos simbólicos que se logran descifrar mediante el recurso al talento de Leonardo. La lectura del Código Da Vinci demanda una incursión en la vida y obra de este gran personaje, lo cual implica conocer de él desde campos tan diversos como sus aportes a la Historia del arte o las implicaciones que tuvo en la historia de la Iglesia. Por no mencionar implicaciones de Da Vinci en otros campos, como la crítica del arte, por la técnica del "sfumato" [esfumado] mencionada en la novela cuando se cometa la pintura de la Mona Lisa o el interés para la arquitectura y la ingeniería que despierta la obra de Da Vinci, conocida como "The Vitruvian Man" [El hombre de Vitruvio], mencionado para descifrar un enigma en la novela.

b) De la segunda parte: El tema de "Hieros Gamos" o prostitución ritual. En el capítulo 74, los personajes centrales discuten e ilustran la práctica ancestral de ese tipo de unión sexual con fines no eróticos sino espirituales por parte de ciertas organizaciones secretas, como el llamado Priorato de Sión, referido en la novela. La intriga que produce este tema en quienes leen, les conmina a investigar acerca de este y se ven involucrados en campos del saber tan variados como la Teología, Sociología y la Antropología (en contextos de simbolismo y religiones comparadas), y el psicoanálisis. Cada una de estas disciplinas le proporciona al lector una amplia visión acerca del fenómeno, con lo cual lo desmitifica y hace desaparecer el tabú. Comprende las razones que las sociedades antiguas tenían para practicarla y las que la teología y tradición cristianas tienen para reprobarla. Con base en esos elementos de juicio, el docente puede motivar a sus estudiantes para que aporten sus propios puntos de vista, mediante el establecimiento crítico de relaciones entre perspectivas tan distintas como complementarias.

En definitiva, el grupo de estudiantes terminan el ejercicio de lectura avanzada con un deseo de que más bien otros cursos de la carrera y otras carreras tuvieran igualmente un enfoque igualmente heurístico, sistémico y ecológico, puesto que quienes aprenden tienen la tarea de realizar una síntesis vital de todo el conocimiento que adquieren, lo cual no es posible si no se les prepara para ello.

Entre las impresiones compartidas por el estudiantado con respecto al pensamiento crítico y la inter y transdisciplinaridad en la educación superior destacan las siguientes:

"El enfoque disciplinario [tradicional] se centra solo en el propio campo de estudios, mientras que el inter, transdiciplinario expande el conocimiento más allá de ese campo." -Karol Chavarría

"Yo recomendaría que se implemente el enfoque inter, transdisciplinario en otros cursos de mi carrera porque nos hace pensar y analizar con mayor profundidad." -Carolina Bosque

"Es importante que los docentes acerquen a sus estudiantes a otras disciplinas, y que tengan en cuenta sus intereses, sus creencias y sus metas durante la carrera." -Melissa Tosso

"En mi carrera, no todos los profesores nos enseñan a aplicar el pensamiento crítico, necesitamos herramientas y estrategias para ello." -Sofía Carvajal 
doi: http://dx.doi.org/10.15359/ree.19-3.22

URL: http://www.una.ac.cr/educare

CORREO: educare@una.cr

\section{La Universidad Nacional de Costa Rica y su reciente reforma de Estatuto Orgánico}

La celebración de un Congreso Universitario para reformar el Estatuto Orgánico refleja la imperiosa necesidad que surge en la institución por ajustarse a las realidades del siglo XXI. Con solo cuatro decenios de existir, la Universidad se sabe inserta en una sociedad de cambios acelerados y servidora de una población cada vez más crítica de las instancias que la gobiernan y de la institucionalidad, en general.

La definición que de sí misma que hace la Universidad en su Preámbulo del Nuevo Estatuto Orgánico, así como los valores, principios y fines que destaca en el Título Primero revelan una voluntad de adecuar no solo sus estructuras, sino también el talante de las personas que la componen a los retos de la Costa Rica del siglo XXI.

En ese sentido, la búsqueda de la complementariedad entre las diversas disciplinas no debe ser vista por la comunidad universitaria como un elemento retórico más que adorna el artículo referido a los fines institucionales. Antes bien, debe entenderse como la condición sine qua non se puede alcanzar ninguna de las demás aspiraciones expresadas en el documento con tan ponderable elocuencia.

Cada Unidad Académica y cada integrante del personal académico, en particular, debe sentir la interpelación de promover esa cosmovisión renovada de la sociedad costarricense, mediante la cual las nuevas generaciones perciban su educación como la instancia que les prepara para que diseñen proyectos de vida, que estén sólidamente articulados y que conduzcan a una genuina realización personal y colectiva.

\section{Conclusión}

En este trabajo se ha intentado poner de manifiesto que no solo es posible, sino, urgente enfocar la formación de las nuevas generaciones de docentes hacia una transformación paradigmática en el ámbito epistemológico.

Se evidenció el hecho de que el paradigma positivista que ha influido en tantas áreas del saber, pero - de manera particular- en la visión de las disciplinas científicas como campos inconexos y que ha llevado a muchos profesionales académicos a radicalizar posiciones de enclaustramiento en sus especializaciones, debe ser sustituido por otro de índole sistémica, que le permita a las nuevas generaciones de profesionales en docencia establecer relaciones entre datos, informaciones y opiniones de diversa naturaleza y que, en un esfuerzo inter y transdisciplinario, realicen un proceso de síntesis vital, lo cual sea una práctica en la organización de sus ideas para que desarrollen y enseñen a desarrollar proyectos de vida coherentes y con propósito. 
El ejemplo planteado en el curso de lectura avanzada en inglés en la Universidad Nacional pretende dar una pincelada del procedimiento metodológico que se puede realizar en la educación superior para alcanzar esos fines. Queda, para futuros análisis y estudios, el planteamiento de nuevas iniciativas para enriquecer a las nuevas generaciones con esta nueva visión de las ciencias y del mundo.

\section{Referencias}

Asencio, E. (2013). Un acercamiento a la formación de docentes de ciencias en Latinoamérica. Experiencias en el contexto cubano. Revista Eureka sobre Enseñanza y Divulgación de las Ciencias, 10 (extraordinario), 797-806. doi 10498/15628

Blázquez, F. (Coordinador). (2001). Sociedad de la información y educación. Mérida: Junta de Extremadura. Recuperado de: http://www.ub.edu/prometheus21/articulos/ obsciberprome/blanquez.pdf

Brown, D. (2004). The Da Vinci Code [El código Da Vinci]. New York: Random House.

Douglas, N., Huntley, H. y Rogers, B. (2012). Reading Explorer 5 [El explorador de lecturas 5]. Boston: Heinle Cengage.

Hahn, K. (Julio, 2003). The Changing Zeitgeist of German Higher Education and the Role of GATS [El Cambiantye espíritu de los tiempos de la educación superior alemana y el papel de las GATS]. Higher Education in Europe, 28(2), 199-215. doi: http://dx.doi. org/10.1080/03797720304096

Lynch, D. R., Russell, J. S., Evans, J. C. y Sutterer, K. G. (Enero, 2009). Beyond the Cognitive: The Affective Domain, Values, and the Achievement of the Vision [Más allá de lo cognitivo: El dominio afectivo, sus valores y el alcance de la visión] Journal of Professional Issues in Engineering Education and Practice, 135(1), 47-56. doi: http://dx.doi.org/10.1061/ (ASCE)1052-3928(2009)135:1(47)

Martínez, M. (Julio-Setiembre, 2009). Hacia una epistemología de la complejidad y transdisciplinariedad. Utopía y Praxis Latinoamericana, 14(46), 11-31. Recuperado de http://www.redalyc.org/articulo.oa?id=27911855003

Martínez, M. (2010). Bases de la epistemología a comienzos del siglo XXI. Revista IIPSI, 13(1), $173-$ 196. Recuperado de http://sisbib.unmsm.edu.pe/bvrevistas/investigacion psicologia/ v13 n1/pdf/a11.pdf 
doi: http://dx.doi.org/10.15359/ree.19-3.22

URL: http://www.una.ac.cr/educare

CORREO: educare@una.cr

Morin, E. (2007). Introducción al pensamiento complejo. Valladolid: Gedisa Editorial. Recuperado de http://www.pensamientocomplejo.com.ar/docs/files/MorinEdgar Introduccion-alpensamiento-complejo Parte1.pdf

Roa, R. (2006). Formación de profesores en el paradigma delacomplejidad.EducaciónyEducadores, 9(1), 149-157. Recuperado de http://www.redalyc.org/articulo.oa?id=83490111

Sadeghi, B., Taghi, M. y Rahmatkhah, M. (Septiembre, 2014). The Relationship between EFL Learners' Metacognitive Strategies, and Their Critical Thinking [La relación entre las estrategias cognitivas de quienes aprenden inglés como lengua extranjera y su pensamiento crítico]. Journal of Language Teaching and Research, 5(5), 1167-1175. doi: http://dx.doi.org/10.4304/j/tr.5.5.1167-1175

Soto, A., Villalobos, I y Villalobos, L. (2007). Guía teórica. Prácticas de comprensión de lectura en inglés II ( $3^{\mathrm{a}}$ reimp.). Heredia: EUNA.

Tudor, I. (2000). The Dynamics of the Language Classroom [La dinámica de la clase de lengua]. Cambridge: Cambridge University Press.

Universidad Nacional de Costa Rica. (2014). Estatuto Orgánico de la Universidad Nacional. Recuperado de http://www.situn.org/index.php/documentos-digitales/finish/10estatutos/31-estatuto-organico-de-la-universidad-nacional

\section{Cómo citar este artículo en APA:}

Barrantes-Montero, L. G. (Setiembre-Diciembre, 2015). Cómo orientar la formación del personal docente nuevo hacia la adquisición de un paradigma sistémico. Revista Electrónica Educare, 19(3), 1-10. doi: http://dx.doi.org/10.15359/ ree.19-3.22

Nota: Para citar este artículo en otros sistemas puede consultar el hipervínculo "Como citar el artículo" en la barra derecha de nuestro sitio web: http://www.revistas.una.ac.cr/index.php/EDUCARE/index 